\title{
Estimation of cyclic plastic deformation behaviours by the micro-indentation method
}

\author{
H. Sakamoto ${ }^{1}$, Y. Ohbuchi ${ }^{1} \&$ Y. Nakamura ${ }^{2}$ \\ ${ }^{1}$ Graduate School of Science and Technology, \\ Kumamoto University, Japan \\ ${ }^{2}$ Osaka Sangyo University, Japan
}

\begin{abstract}
The change of mechanical properties under cyclic loading was measured and the cyclic hardening/softening phenomena at the early stage of fatigue around the crack tip were made clear by the Micro-Indentation Method. The effects of the stress concentration, the strain amplitudes and the number of cycles on the dynamic micro hardness of the material surface were examined in detail. The cyclic local plastic behaviours agree well with the change of surface micro dynamic hardness and it is shown that this Micro-Indentation Method is effective as the evaluation method of mechanical properties under cyclic loading.
\end{abstract}

Keywords: plastic behaviour, micro-indentation, cyclic loading, plastic hardening, low cycle fatigue.

\section{Introduction}

Recently, as the evaluation method of the mechanical properties of surface treatment material and thin film, the Micro-Indentation Tester has received attention from micro-electron manufacturing industries [1-4]. The change of material properties under cyclic loading plays an important role in the fatigue process. In this study, the change of material properties, that is, the cyclic hardening/softening phenomenon at the early stage of fatigue around the crack tip, was measured by a Micro Indentation Tester [5]. The effects of the stress concentration, the strain amplitudes and the number of cycles on the surface micro hardness were examined. The conventional method of micro Vickers hardness demands the measurement of the diagonal length of indentation and a lot of time is required for this measurement. This report shows that the Micro- 
Indentation Method can measure the indentation depth as "dynamic hardness" of a material's surface in an automatic and short time and this method is effective for evaluating cyclic plastic behaviours.

\section{Experiment}

\subsection{Material and specimen}

The material used is austenite stainless steel plate (thickness 6mm), SUS304 in JIS. The crystal grain sizes were $50 \sim 200 \mu \mathrm{m}$. The material properties were obtained by a monotonic tensile test and a cyclic strain test. Figures 1(a) and (b) show the monotonic stress-strain curves and the cyclic stress-strain curves obtained by connecting the top points of stabilized hysteresis loops [6]. This material cyclically softens in a small strain region $(<0.4 \%)$ and cyclically hardens under larger strain than that, and this material shows the strain rate dependence at room temperature.

Figure 2 shows the geometry and dimensions of hardness in the measured specimen. The centre pre-crack was made by an electro spark machine with wire of $0.03 \mathrm{~mm}$ diameter as stress concentration parts. All specimens were annealed (in vacuum, $800^{\circ} \mathrm{C} 3.0 \mathrm{hr}$ hold, furnace cooling) and the solution heat treatment (in vacuum, $1030^{\circ} \mathrm{C} 2.0 \mathrm{hr}$ hold, rapid cooling) was made.

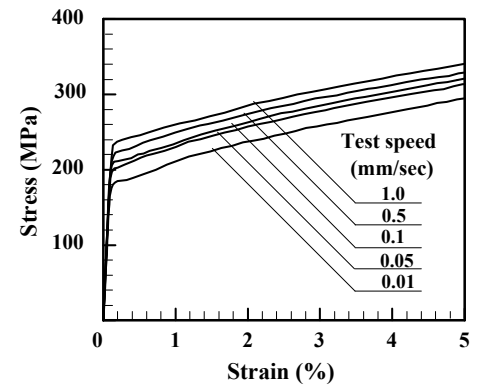

(a) Monotonic Stress-strain curves

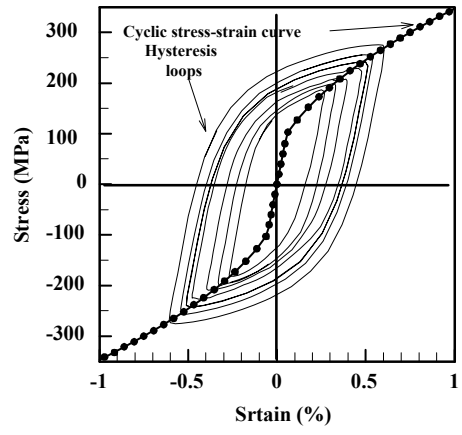

(b) Cyclic Stress-strain curves

Figure 1: Monotonic and cyclic stress-strain curves.
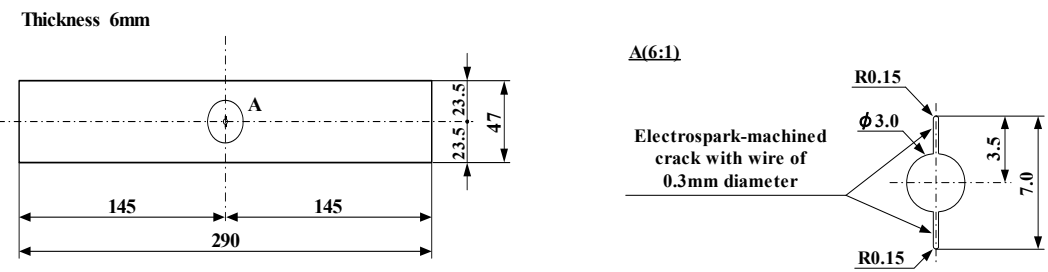

Figure 2: $\quad$ Test specimen. 


\subsection{Experimental procedure}

\subsubsection{Cyclic loading test}

In order to examine the change of material properties under cyclic loading, low cycle fatigue tests were carried out by displacement control; the displacement amplitudes are $0.25 \mathrm{~mm}, 0.3 \mathrm{~mm}, 0.35 \mathrm{~mm}$ and the subjected number of cycles are 10, 50, 100, 1000 at each amplitude.

The strain behaviours were measured by strain gauges that were pasted at $1.5 \mathrm{~mm}$ distance from the crack tip. In this cyclic loading test, an electrohydraulic servo-type fatigue-testing machine was used.

\subsubsection{Dynamic micro hardness test}

The surfaces of the specimens obtained by the cyclic loading test were treated by electrolytic polishing. Surface roughness is about $0.04 \mu \mathrm{m}$ in Ra. In this hardness test of the material surface, Dynamic Ultra-Micro Hardness Tester (DHU-201S, Shimadzu co.) was used. Figure 3 shows the effect of the loading speeds and the loading weight of the Vickers hardness (Hv) in raw material. The surface hardness obtained by the light weighing shows higher than that by heavy weighting, but each measurement value in the same loading speed is in a narrow band. In this study, the hardness tests were carried out for each specimen in the load $294 \mathrm{mN}$ and $10 \mathrm{sec}$ holding time, and Vickers indenter was used in order to compare with conventional Vickers hardness

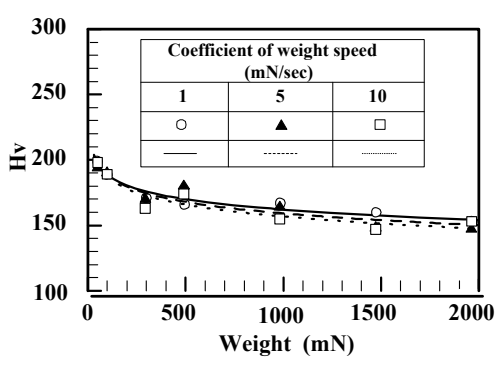

Figure 3: $\quad$ Effect of loading speeds.

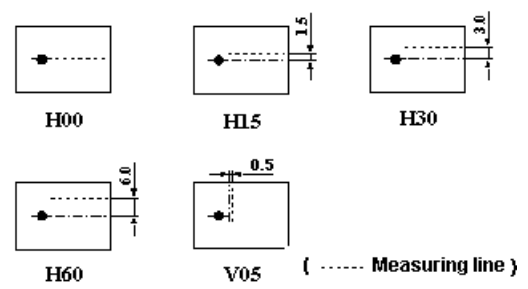

Figure 4: $\quad$ Measuring positions of hardness. 
Figure 4 shows the measuring lines around the crack tip, H00,H15,H30,V05; the measuring points of each line is 90 points. The hardness of each point was evaluated by mean value of 3 points and the dynamic Vickers hardness was calculated from the load and the indentation depth.

\section{Results and discussion}

The hardness distribution after 10 cycles and 1000 cycles along line H00 are shown in Figs. 5(a) and (b), respectively. The hardness along the crack line increase at the vicinity of the crack tip due to the cyclic plastic deformation by the stress concentration shown in Fig. 5(a). Increasing the number of cycles, the tendency becomes remarkable by development of cyclic plastic deformation. Figure 6 shows the change of hardness distributions along each measured line. From this figure, it is found that the hardness largely increases in the early stage of fatigue.

Figure 7 shows the change of the crystal grain size which was obtained by etching schematically and the martensitic structure was observed at this cyclic plastic region around crack tip.
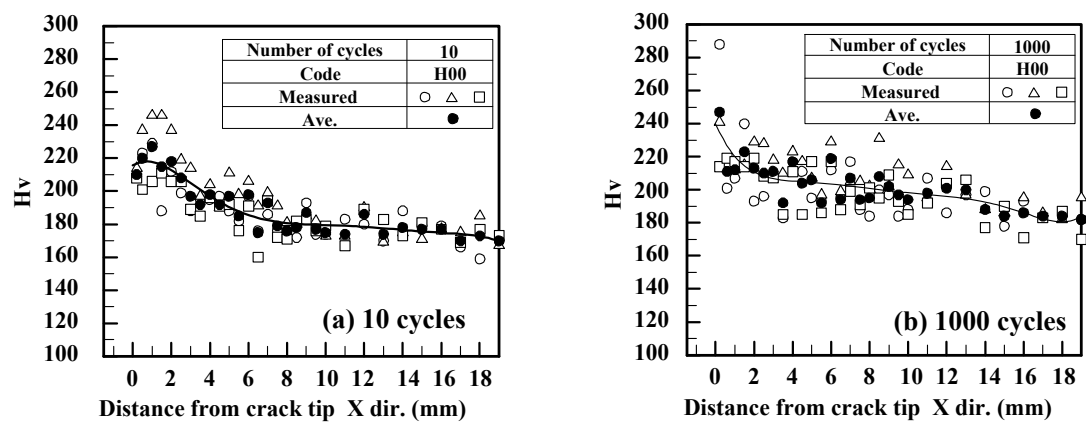

Figure 5: $\quad$ Hardness distribution along line H00.

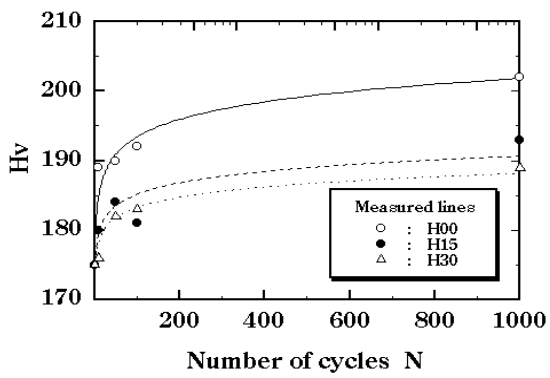

Figure 6: Change of hardness distribution at each line. 

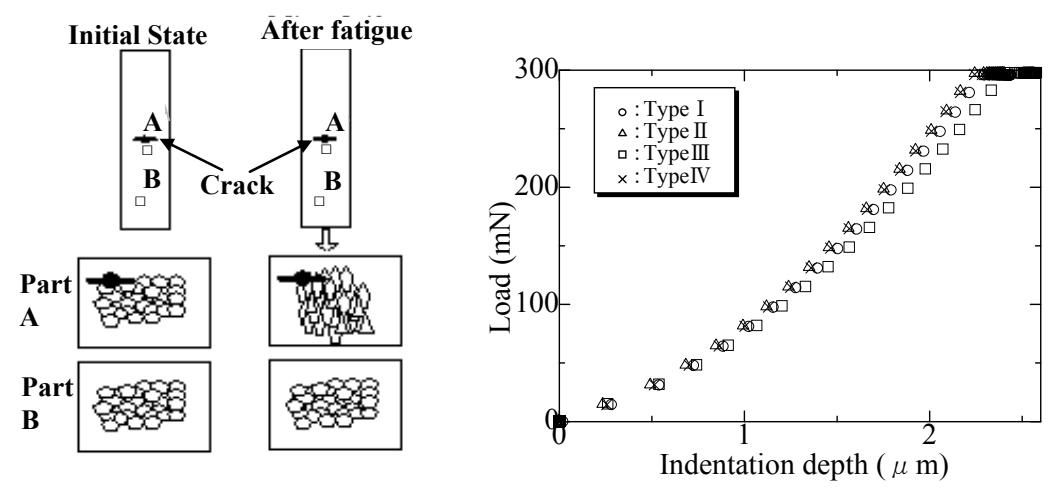

Figure 7: Change of crystal size after fatigue.

Figure 8: Load-indentation depth curves.
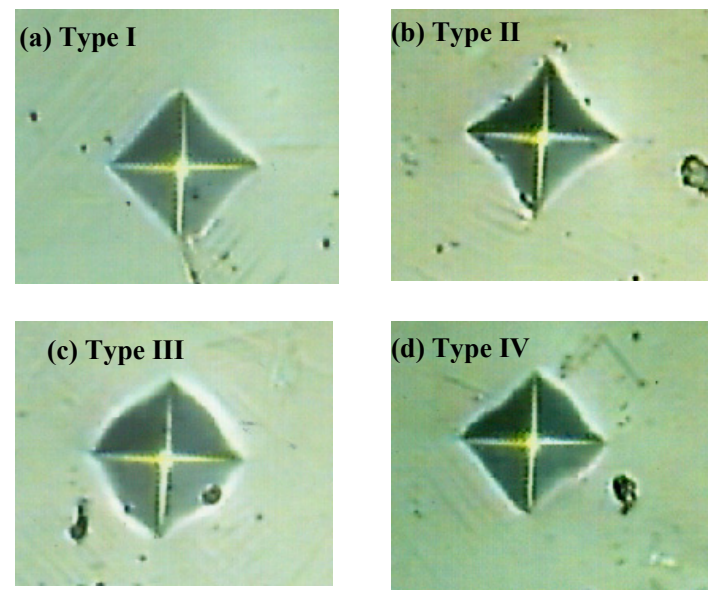

Figure 9: Configuration of indentation.

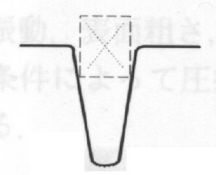

(a) Type I

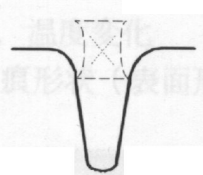

(b) Type II

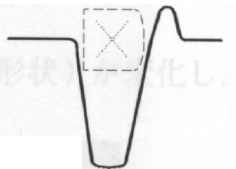

(c) Type III

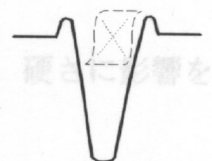

(d) Type IV

Figure 10: Configuration of cross section of indentation. 
Next, we examined the relation between the load-indentation depth curve and the configuration of indentation. The configurations of indentation were classified into four types shown in Fig.9 and the load-indentation depth curves were shown in Fig.8. It becomes soft in the order of Type IV, type II, Type I, Type III. The corresponding configurations of the cross section of indentation are shown in Fig.10. From these figures, it is found that the change of mechanical properties corresponded to the change of micro hardness.

\section{Conclusions}

The change of material property under cyclic load was evaluated by the change of dynamic micro hardness of material's surface and indentation shape. The results obtained are summarized as follows:

1) The hardness in vicinity of crack tip increase rapidly due to stress concentration and the hardness distribution correspond well to the strain distribution

2) Dynamic hardness measured by indentation depth corresponds well to conventional Micro Vickers hardness. So, the material property can be evaluated speedy by using Dynamic Ultra Micro Hardness Tester.

\section{References}

[1] Doerner, M.F. \& Nix, W.D., A method for interpreting the data from depthsensing indentation instruments, Journal of Materials Research, 1-4, pp.601-609, 1986.

[2] Oharr, G.M. and Oliver, W.C., Measurement of thin film mechanical properties using Microindentation, MRS Bulletin, 7, pp.28-33, 1992.

[3] Yasuda, K. Shinohara, K., Yamada, M., Kutsuwada, M. \& Kinoshita, C.,A New method for evaluating stress-strain properties of metals using ultra microhardness technique, Journal of Nuclear Materials, 187, pp.109-116, 1992.

[4] Weiler, W., Hardness Testing - a new method for economical and physically meaningful microhardness testing, British Journal of NDT, 31, p.253, 1995.

[5] Yamamoto, Y. \& Nishimura T., Functions and applications of Shimadzu's DUH-201 and DUH-201S Dynamic Ultra-Microhardness Tester, Shimadzu Technical Report, 50-3,pp.321-329,1993

[6] Landgarf, R.W., Morrow, J.A \& Endo, T., Determination of the cyclic stress-strain curve, Journal of Materials, JMLSA, 4-1, pp.176-188, 1969. 